\title{
Mudanças climáticas globais: Viés de percepção, tempo e espaço
}

José Q. Pinheiro. Universidade Federal do Rio Grande do Norte.

Giselli R. C. Cavalcanti. Universidade Federal do Rio Grande do Norte.

Hellen C. L. Barros. Universidade Potiguar.

\section{Resumo}

Investigamos a percepção das mudanças climáticas globais e de sua gravidade em relação às escalas espacial e temporal. Colaboraram 52 estudantes, de ensino médio e superior, sendo 17 do sexo feminino e 35 do masculino. Utilizamos um questionário com perguntas tanto abertas como de múltipla escolha. Detectamos paralelos com o viés de otimismo espacial reportado na literatura, ou seja, uma visão mais positiva da situação quando se trata de ambientes geograficamente mais próximos do respondente. Esse otimismo também foi claramente identificado na escala temporal do momento presente. Para temporalidades maiores (10, 25, ou mais anos no futuro), as tendências observadas não foram tão claras, mas sugerem um otimismo temporal, e não o pessimismo temporal ("as coisas vão piorar") encontrado em estudos anteriores. Esses achados podem nortear projetos de educação ambiental que enfoquem o âmbito local e condições de existência no mesmo, indo além da avaliação positiva costumeiramente identificada na literatura.

Palavras-chave: mudanças climáticas globais; percepção ambiental; escala espacial; escala temporal; mitigação.

\section{Abstract}

Global climate changes: Perception bias, time and space. We investigated the perception of global climate changes (GCCs) and their severity in relation to spatial and temporal scales. Participants were 52 students of secondary and higher education, being 17 females and 35 males. We employed a questionnaire containing open- ended and multiple-choice issues. We detected parallels with the bias of spatial optimism reported in the literature, that is, a more positive assessment of the situation for environments geographically closer to the respondent. This optimism was also clearly identified in the time scale of the present moment. For major temporalities (10, 25, or more years in the future), the trends observed were not so clear, but suggest a temporal optimism, not the temporal pessimism ("things will get worse") found in previous studies. These findings can guide environmental education projects that focus on the local scope and conditions of existence in the same, going beyond the positive evaluation customarily identified in the literature.

Keywords: global climate change; environmental perception; spatial scale; temporal scale; mitigation.

\section{Resumen}

Cambio climático global: Sesgo de percepción, tiempo y espacio. Investigamos la percepción de los cambios climáticos globales (CCGs) y de su gravedad con relación a las escalas espacial y temporal. Colaboraran 52 estudiantes, de enseñanza media y superior, siendo 17 del sexo femenino e 35 del masculino. Utilizamos un cuestionario con preguntas abiertas y de elección múltiple. Detectamos paralelos con el sesgo de optimismo espacial mencionado en la literatura, o sea, una visión más positiva de la situación cuando se trata de ambientes geográficamente más cercanos del respondiente. Ese optimismo también fue claramente identificado en la escala temporal del momento presente. Para temporalidades mayores (10, 25, o más años en el futuro), las tendencias observadas no fueran tan claras, pero sugieren un optimismo temporal, y no el pesimismo temporal ("las cosas van a empeorar") encontrado en estudios anteriores. Estos hallazgos pueden orientar proyectos de educación ambiental que enfoquen el ámbito local y condiciones de existencia en el mismo, yendo más allá de la evaluación positiva habitualmente identificada en la literatura.

Palabras clave: cambio climático global; percepción ambiental; escala espacial; escala temporal; mitigación. 
Desde a Revolução Industrial, vivemos com base em um modelo de desenvolvimento em que se emprega o uso dos recursos de forma pouco solidária e pouco sustentável (Held, 2001; Intergovernmental Panel On Climate Change [IPCC], 2014). Estes ideais levaram a um agravamento do chamado efeito estufa, acentuado principalmente pelo aumento da concentração na atmosfera dos gases do efeito estufa (GEEs), como o dióxido de carbono $\left(\mathrm{CO}_{2}\right)$ e o metano $\left(\mathrm{CH}_{4}\right)$, emitidos, em grande parte, pela intensificação da atividade humana no planeta (IPCC, 2014). O quinto relatório de avaliação do Painel Intergovernamental sobre Mudanças Climáticas (ou IPCC, sua conhecida sigla em inglês), publicado em 2014, apontou que houve um aumento em torno de $0,85^{\circ} \mathrm{C}$ até o ano de 2012 .

Os impactos deste aquecimento são globais, intensos e variados, ocasionam mudanças nos padrões climáticos, aumentam a ocorrência de eventos extremos, alteram o padrão de chuvas e secas das regiões, aumentam o derretimento de geleiras e os níveis dos oceanos. As consequências e impactos das mudanças climáticas globais (MCGs) para a qualidade dos ecossistemas são, então, inúmeros, e acarretam outros impactos que vão afetar mais diretamente a vida humana, em uma cadeia complexa de acontecimentos (IPCC, 2014).

No cenário brasileiro, há o Painel Brasileiro de Mudanças Climáticas (PBMC), cujo objetivo é fornecer avaliações científicas sobre as MCGs de relevância para o território nacional, subsidiando o processo de formulação de políticas públicas e tomada de decisão para o enfrentamento dos impactos dessas mudanças, servindo também como fonte de informações de referência para sociedade (PBMC, 2014a; 2014b; 2014c). Várias projeções de impactos já foram apresentadas para o Brasil, sendo diversas as regiões e os setores da economia afetados. Antecipa-se que na agricultura, por exemplo, grandes áreas de plantio serão perdidas; aumentará o risco de extinção de espécies; serão acentuadas a erosão costeira e as mudanças nos padrões pluviais; na área da saúde, além do efeito direto do estresse por calor, de problemas respiratórios e consequente risco para mortalidade, o ambiente terá condições mais favoráveis para a disseminação do mosquito Aedes aegypti (Nobre et al., 2016; PBMC, 2014b). Além disso, a própria geração de energia poderá sofrer perdas. "As fontes renováveis de energia representam, de um lado, uma alternativa para a mitigação da mudança do clima global. De outro, por serem dependentes das condições climáticas, estão potencialmente sujeitas a impactos do próprio fenômeno que pretendem evitar" (Rosa, 2008, p. 7).

Seja combatendo as causas do problema por meio de ações de mitigação (amenização), ou proporcionando medidas de adaptação de comunidades atingidas pelas consequências do mesmo, a ação humana está diretamente envolvida, assim como instâncias a ela associadas: psicológicas, comportamentais, sociais, político-econômicas e culturais (Clayton et al., 2015; Gifford, 2008; Moser \& Dilling, 2006; O'Neill, 2008; Pawlik, 1991; Stern, Young, \& Druckman, 1992; Swim et al., 2009; Uzzell, 2000; Whitmarsh, O'Neill, \& Lorenzoni, 2011). Foi, principalmente, a partir do início da década de 1990, que estudos passaram a destacar o papel humano diante de problemáticas ambientais globais (Pawlik, 1991; Stern et al., 1992; Uzzel, 2000), sendo este um fenômeno de investigação necessariamente interdisciplinar, por envolver as instâncias humanas mencionadas, incluindo sua abordagem pela Psicologia (Barros \& Pinheiro, 2013; Clayton et al., 2015; Doherty \& Clayton, 2011; Gifford et al., 2009; Lima, 2009; Stern, 2011; Swim et al., 2009).

Investigar os aspectos psicológicos envolvidos nas mudanças climáticas e as consequentes ações de âmbito local e global pode favorecer o incentivo a comportamentos pró-ecológicos, de adaptação e de mitigação/amenização do problema. Pode estimular a aceitação de políticas públicas que busquem combater as MCGs, ou ainda, pode favorecer a cobrança individual e comunitária para que tais políticas possam ser pensadas, e o atual padrão de exploração ambiental possa ser continuamente questionado (Gifford, 2008). A esfera de atuação diante das MCGs pode e deve ser tanto individual como coletiva.

Atualmente, três grandes áreas de investigação sobre aspectos psicológicos e MCGs podem ser citadas; são elas: a) comunicação e percepção das MCGs; b) comportamentos de mitigação e de intensificação do problema e seus preditores; e c) comportamentos de adaptação, sentimentos, e bem-estar frente aos seus impactos. Futuras pesquisas podem se pautar por uma ou mais destas esferas, sempre levando em conta como vieses na percepção das escalas espacial e temporal podem afetar as respostas ao problema (Clayton et al., 2015; Gifford et al., 2009; Uzzell, 2000).

\section{Percepção das Mudanças Climáticas Globais e o Envolvimento em sua Mitigação}

A experiência direta das MCGs pelas pessoas é improvável, até impossível, pois suas alterações são 
imperceptíveis, por exemplo, quando comparadas às mudanças sazonais do próprio ano (Pawlik, 1991; Uzzel, 2000). Também não há uma relação clara e precisa entre suas causas e consequências, e, além disso, essas mudanças ocorrem em todo o planeta, sendo distintas de um problema mais pontual e concreto como um cano de água estourado em nossa rua (Barros \& Pinheiro, 2013). Tais peculiaridades tornam as MCGs um problema complexo, de caráter abstrato e de apreensão intrincada, o que favorece a existência de barreiras psicológicas para ações de mitigação/ atenuação e de adaptação às suas consequências (Gifford, 2011). "Aquecimento global", ou "mudanças climáticas globais" já se tornaram moda; são expressões presentes em todas as formas e meios de comunicação. No entanto, sua difusão descontrolada pela mídia não assegura sua devida apropriação/ decodificação. A começar pelo próprio adjetivo global. Quantos dos que são expostos a essas expressões têm uma ideia bem acabada do que está aí contido? Do que é o globo?

Algumas das medidas de mitigação apontadas pelos relatórios científicos no contexto brasileiro são: a) a redução de carbono, por meio da promoção e recuperação de áreas que foram degradadas para pastagem; b) a mudança no padrão pecuarista, com encorajamento de adoção de sistemas lavoura-pecuária; c) a substituição dos combustíveis fósseis usados pela indústria, com a geração de energia solar e eólica; d) a implementação de sistemas de transporte públicos integrados e mais eficientes, com a ampliação de linhas de metrô e trens urbanos, implantação de linhas de veículos leves sobre trilho, e a implementação de medidas de gerenciamento de tráfego. Além disso, a redução de veículos automotivos nas áreas urbanas é extremamente necessária, assim como é necessário frear o desmatamento na Amazônia (PBMC, 2014b; 2014c).

Essas ações são do âmbito da gestão pública, mas outras ações individuais e coletivas também são demandadas. Desde o trabalho comunitário para cobrança destas medidas, aceitação das mesmas quando implantadas, e mudanças nos padrões de transporte, e de consumo energético, até a promoção de plantio e manutenção de áreas verdes, realização de um consumo consciente, reutilização e engajamento em reciclagem. Todas essas medidas contribuem direta ou indiretamente para reduzir a manutenção de GEEs na atmosfera, e consequentemente mitigam as mudanças climáticas globais (PBMC, 2014c).
O papel institucional, de gestão e de políticas públicas é, então, extremamente relevante para a adesão a comportamentos individuais de mitigação, e muitos estudos discutem este papel, com aspectos tecnológicos e econômicos sendo levados em conta. As condições macro-políticas e interesses internacionais também influenciam fortemente nas medidas ambientais globais adotadas, influências que são pautadas em uma arena de conflitos multilaterais, na qual países discordam sobre seus papéis na ocorrência do problema e em suas responsabilidades para mitigação. Tais conflitos atrasam e dificultam negociações, se tornando extremamente prejudiciais para o futuro da qualidade de vida humana no planeta (IPCC, 2014).

Todavia, fatores individuais têm recebido menos atenção. É relevante prestar atenção a estes aspectos comportamentais individuais já que são eles que estarão relacionados à adoção de novas tecnologias, e que darão suporte, ou não, às políticas traçadas de enfrentamento (Clayton et al., 2015). Ao pensar a esfera individual, reconhece-se que esta representa um componente neste complexo cenário multifacetado de relações político-sociais. É preciso que a percepção individual do fenômeno seja compreendida dentro de uma esfera macro, que vai além do indivíduo por si só. Nesse sentido, compreende-se que essa percepção é diretamente influenciada por valores, atitudes, influencias sociais e identidade cultural, como mostram os estudos sobre percepção de risco aplicada às MCGs, que incluem processos sociais e culturais que podem levar à amplificação ou atenuação desse risco (Breakwell, 2010; Leiserowitz. 2005; Lorenzoni, Pidgeon, \& O'Connor, 2005; Renn, 2011).

Whitmarsh (2009), entre outros autores, interessou-se por investigar quais ações as pessoas estavam adotando especificamente para mitigar as MCGs, e quais os principais determinantes dessas ações. Os resultados de sua investigação apontaram que poucas pessoas se engajavam em comportamentos de mitigação de forma intencional, ou seja, com o intuito deliberado de mitigar; as pessoas que economizavam energia - ação estimulada pelo governo - o faziam por benefícios no pagamento da conta de energia e não para amenizar as MCGs. Já as pessoas que buscavam mitigá-las deliberadamente optavam por outros tipos de ação, que não apenas economia energética, sendo a reciclagem uma das mais mencionadas nesses casos. Convém também mencionar que passar de atitudes e motivações para comportamentos concretos é um salto que 
ainda traz dificuldades mesmo para pessoas bem intencionadas, com alto grau de informação e convictas da necessidade desses comportamentos de mitigação, dificuldade essa que pode advir de fatores econômicos ou sociais (Renn, 2011).

É importante considerar que as ações em prol do enfrentamento do problema podem ser extremamente diversificadas; algumas podem ser mais diretamente efetivas no combate às MCGs, enquanto outras podem auxiliar de forma mais indireta (PBMC, 2014c). Algumas podem ser melhor compreendidas e de fácil execução, outras podem ser mais complexas e não serem conhecidas ou visualizadas pelas pessoas (Whitmarsh, 2009), aspectos diretamente relacionados às dimensões temporal e espacial da percepção das MCGs.

\section{As Escalas Temporal e Espacial na Percepção das Mcgs}

As mudanças climáticas globais correspondem a um problema de fronteiras de espaço e de tempo muito difíceis de visualizar. Por isso, elas forçam a que se reflita sobre a relação local-global (Feitelson, 1991) e sobre como as pessoas se situam nessa relação, já que se está falando de um problema do globo que afeta as localidades e é causado nelas. A interação entre o local e o global parece ser crucial ao entendimento das percepções públicas e atitudes em relação aos problemas ambientais globais e aos comportamentos relacionados (Uzzell, 2000). Nessa direção, algumas investigações têm buscado se apropriar deste debate. Um estudo realizado em parceria com diversos países (Gifford et al., 2009), mostrou a tendência de as pessoas avaliarem de forma mais positiva os problemas ambientais de suas localidades, e de forma mais negativa os mesmos problemas quando se trata do mundo, evidenciando uma negação ou atenuação dos problemas no âmbito local (otimismo espacial).

No caso das MCGs, essa negação se refere a uma avaliação cognitivo-afetiva de não existência do problema e de seus impactos para os níveis locais (Doherty \& Clayton, 2011). Essa avaliação dificulta comportamentos em prol do meio ambiente em ambos os níveis espaciais. Pois, quando se trata da localidade, a situação é avaliada como boa, não sendo preciso fazer nada; quando se trata do globo, a ideia é de que está ruim, mas uma ação individual é pequena demais para resolver alguma coisa (Gifford et al., 2009).

Nesse sentido, estaríamos diante da crença generalizada de que eventos indesejáveis são menos prováveis de acontecer a si mesmos, e mais prováveis de acontecer aos demais, o que constituiria o viés do otimismo (Barros \& Pinheiro, 2013; Gifford, 2011; Gifford et al., 2009; Uzzell, 2000). Clayton et al (2015) retomam esse viés do otimismo em relação às MCGs, destacando que o problema pode ser negado ou rejeitado, caso as pessoas sintam seus estilos de vida ameaçados por novas propostas governamentais para o enfrentamento da situação. Assim, levar em conta as escalas espaciais, local-global, tem sido um passo fortemente sugerido, como algo que pode contribuir para a maneira como as mudanças climáticas são percebidas (Clayton et al., 2015; Feitelson, 1991; Scannell \& Gifford, 2013; Uzzell, 2000).

Os estudos psicológicos das MCGs também devem levar em conta a escala temporal (hoje-futuro). Por influência dela, as pessoas percebem e dão significado ao mundo que as rodeia. Além disso, não se pode entender o espaço e o lugar sem levar em consideração a história das pessoas e suas capacidades de se projetar para o futuro (Moser \& Uzzel, 2004), sobretudo quando se fala em MCGs. Afinal, suas consequências começam a ocorrer depois de um longo período de tempo após a ocorrência de suas causas, o que faz com que a emissão de comportamentos de mitigação possa estar relacionada a uma orientação de futuro da pessoa.

Uma avaliação positiva consideraria a situação futura como sendo mais favorável do que a atual. A pesquisa já mencionada sobre percepção de problemas ambientais, entretanto, constatou pessimismo temporal, ou seja, os problemas eram percebidos como piores no futuro, independentemente da escala espacial considerada (Gifford et al., 2009).

As MCGs envolvem, então, escalas que ultrapassam o tempo do agora e às redondezas geográficas dos indivíduos. Compreender o papel dessas escalas pode ser um caminho promissor para entender a percepção ambiental que se tem do problema e, desse modo, aprimorar formas de comunicá-lo. Assim, os objetivos estabelecidos para este estudo foram investigar: a) quais as ações de mitigação das MCGs seriam indicadas pelos respondentes; b) a percepção da gravidade das MCGs de acordo com variações nos níveis da escala espacial; e c) a percepção de quando a ameaça das MCGs se materializará nos cenários geográficos propostos.

\section{Método}

\section{Participantes}

Este estudo descritivo-exploratório foi realizado com um total de 52 alunos de duas escolas do Rio Grande do Norte. Uma das escolas é pública (IFRN), localizada em 
cidade do interior do estado (João Câmara) em que vários parques eólicos foram construídos nos últimos anos; dos seus 28 alunos, 12 eram do Curso de Eletrotécnica (ensino médio, com idade média de 17 anos) e 16 do Curso Técnico Superior em Energias Renováveis (média de idade de 24 anos). Os demais 24 alunos frequentavam o nono ano do ensino fundamental na outra escola, particular, localizada na capital do estado, tendo média de idade de 14 anos. Do número total de participantes, 17 eram do sexo feminino e 35 do masculino.

A escolha por um grupo de participantes que estivessem matriculados em um curso sobre Energias Renováveis se deu, especialmente, por haver o pressuposto de que tais estudantes teriam, tanto na sua constituição pessoal (o que os motivou a escolher o curso) quanto na sua formação escolar, um maior direcionamento para as temáticas investigadas neste estudo. Em comparação a estes participantes, achamos relevante ter também um grupo que não estivesse inserido no curso de Energias Renováveis, mas que também fizesse parte deste contexto maior (mesma instituição de ensino e mesma cidade), o que nos levou a escolher os alunos do curso de Eletrotécnica. Para o terceiro grupo, o critério de escolha foi tentar perceber como os adolescentes mais novos interagem com essa temática e com o questionário desenvolvido por nós, considerando o valor heurístico deste estudo para investigações na área.

\section{Instrumentos e Procedimentos}

Construímos um questionário contendo perguntas relacionadas aos dados sócio-demográficos, tais como: idade, sexo, e grau de instrução da mãe e do pai. Em seguida, vinham as questões do estudo a fazer referência direta à gravidade da ameaça contida nas MCGs, que foram elaboradas a partir de uma adaptação do estudo de O'Neill (2008).

Buscávamos explorar a percepção da gravidade do problema em função de duas condições: o quanto os respondentes estimavam que essa ameaça era grave para cada escala espacial ("Selecione, com base nas opções do quadro abaixo - seu bairro; sua cidade; o Brasil; o mundo todo/ Terra -, quanto você sente que é grave - não é grave, não muito grave, grave, muito grave - a ameaça das mudanças climáticas globais"); e quando ela passaria a ser grave para cada um daqueles ambientes ("Com base no quadro abaixo - seu bairro; sua cidade; o Brasil; o mundo todo/ Terra -, selecione quando você acha que as mudanças climáticas globais são, ou passarão a ser, perigosas - agora, em 10 anos, em 25 anos, em 50 anos, em 100 anos, nunca").
Por fim, em questão inspirada em Barros e Pinheiro (2013), pedimos para que o participante se colocasse, de forma imaginativa, em uma situação na qual estaria conversando com um colega sobre as formas de enfrentar as MCGs e, a partir disso, ele deveria indicar atividades/ações adequadas para esse objetivo.

O questionário foi aplicado aos participantes em horário de aula, disponibilizado pelas coordenações dos cursos e professores, que previamente aprovaram a realização da pesquisa, respeitados os aspectos éticos pertinentes.

\section{Análise dos dados}

As respostas à questão aberta, relativa às formas de mitigação/ amenização das MCGS, foram transcritas e analisadas com base em análise temática de conteúdo (Bardin, 1977; Bauer, 2002; Castro, Abs, \& Sarriera, 2011). Foram criadas categorias representativas das ideias centrais das respostas, as quais foram posteriormente implantadas em planilha eletrônica. A análise das questões numéricas envolveu estatísticas de tendência central, frequências, testes de qui-quadrado e comparação de médias.

\section{Resultados e discussão}

Os resultados obtidos são expostos e discutidos em blocos temáticos, de acordo com os objetivos propostos para a investigação. Não identificamos nos dados obtidos quaisquer diferenciações estatisticamente significativas no que diz respeito aos dados sociodemográficos examinados, a saber: idade, sexo e grau de instrução da mãe e do pai. Iniciamos a seção com o bloco temático sobre ações de mitigação das mudanças climáticas globais (MCGs).

\section{Posicionamento do Respondente sobre Ações de Mitigação das Mcgs}

A partir das respostas dadas em relação ao que poderia ser feito como ação de combate frente às MCGs, foram criadas categorias, nas quais pudessem ser representadas as ideias centrais que estavam ali contidas, conforme discriminação na Figura 1. Não dispomos neste artigo do espaço necessário para apresentar exemplos de respostas dos participantes para cada categoria; o leitor interessado nesse detalhamento pode consultar o trabalho de Barros (2011), no qual a presente categorização foi baseada. 


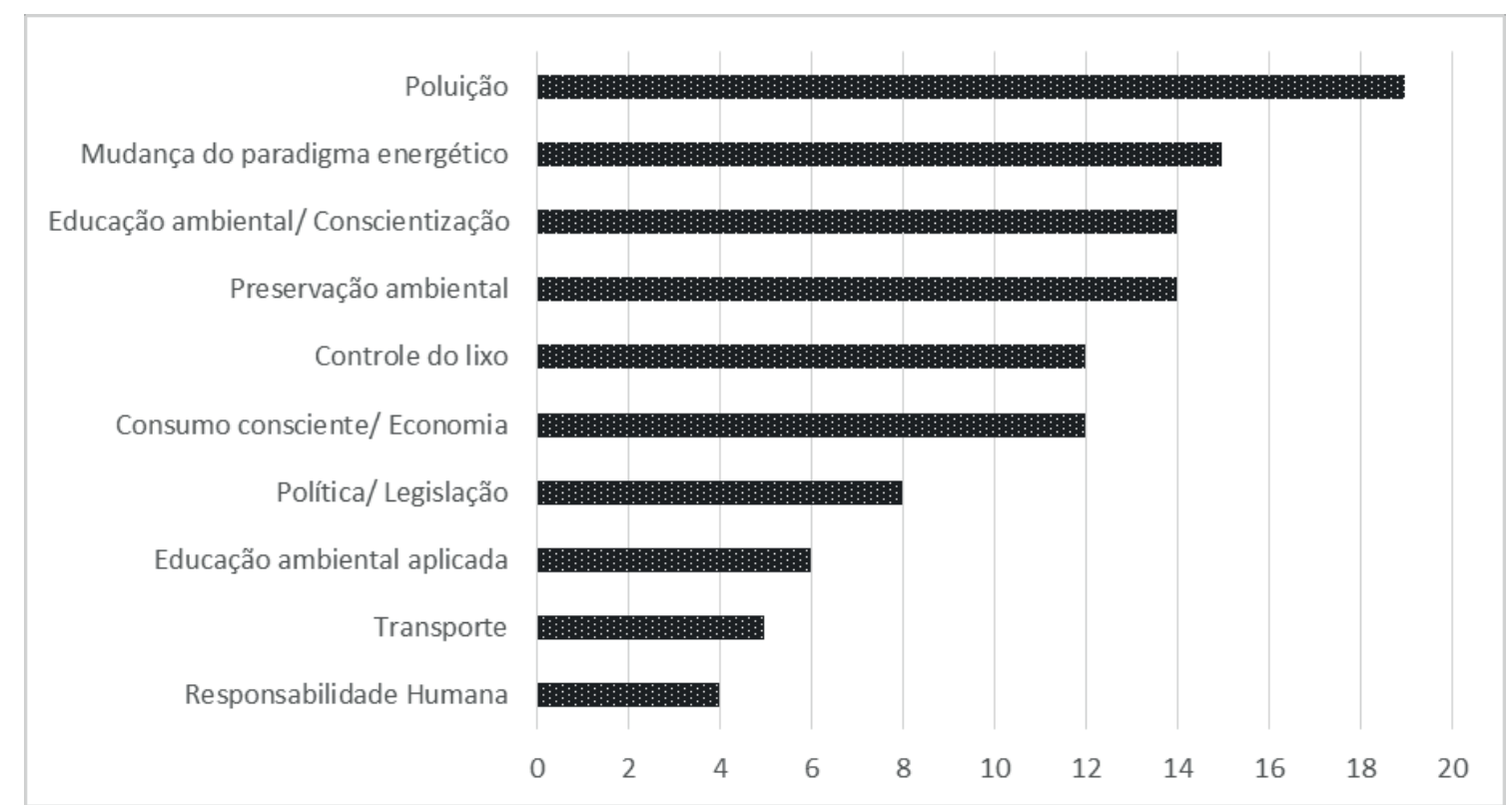

Figura 1. Frequências percentuais das ações de mitigação das MCGs mencionadas pelos participantes (total de menções = 104).

Com base nessas categorias e em sua frequência percentual, constatamos que as MCGs ainda parecem ser um conceito confuso para os respondentes, dada a sua alta associação com concepções tradicionais de problemas ambientais como poluição ( $20 \%$ do total de 104 menções), preservação ambiental (12\%) e controle do lixo (11\%), por exemplo. Estas são categorias que apesar de estarem relacionadas às mudanças climáticas, são fatores secundários, constatação que também havia sido observada no estudo de Barros e Pinheiro (2013).

Por outro lado, é importante destacar a categoria mudança do paradigma energético (14\%), que foi a segunda ação de mitigação mais mencionada pelos participantes e é, de acordo com a literatura (IPCC, 2014), a principal forma de combate que se deve adotar hoje. Convém ressaltar que quase a metade dessas menções (44\%; ou sete, do total de 16) foi apresentada pelos alunos do curso de Técnico em Energias Renováveis, ocorrendo em frequência observada ( $f o$ ) estatisticamente superior à esperada ( $f e$ ) ( $f o=7>f e=$ $4,6)$, o que possivelmente expressa um viés decorrente da especialidade desse subgrupo.

Além disso, duas ações trazidas pela literatura da área como práticas eficazes de mitigação foram também encontradas nas respostas dadas. A categoria de consumo consciente faz referência ao consumismo desenfreado como uma das causas do problema e a necessidade de mudar esse estilo de vida, coincidindo com recomendações da literatura (e.g., Corral-Verdugo, 2010). A segunda delas, referente ao transporte, abarca as respostas que traziam a necessidade da mudança no modelo tradicional de locomoção (transporte particular, baseado em combustíveis fósseis). Esta categoria está em conformidade com o relatório do IPCC, de 2014, que indica o modelo atual de transporte como sendo responsável por $11 \%$ do aumento das emissões de gases poluentes e como um dos focos para as ações de mitigação.

Ainda que categorias relacionadas a aspectos secundários das MCGs (como poluição e preservação ambiental) tenham sido encontradas nas respostas dos participantes com altas frequências, a menção às três ações discutidas anteriormente (mudança no paradigma energético, consumo consciente e transporte) pode indicar uma compreensão mais abrangente e adequada da questão das MCGs por alguns dos participantes. Ações mais diretamente vinculadas às mudanças climáticas, e tradicionalmente não encontradas em estudos da área em nosso meio, possivelmente sinalizam uma visão mais bem informada da parte de alguns dos respondentes.

Sobre uma possível diferenciação pelos alunos do Curso de Técnico em Energias Renováveis, além da maior menção da categoria de mudança do paradigma energético, como já sinalizado, também houve destaque ( $f o=5>f e=1,8 ; p=0,011$ ) para educação ambiental 
aplicada (ações como participação de mutirões de limpeza, de grupos de pesquisa, cursos e palestras) como uma ação de mitigação eficaz. Esse destaque pode estar relacionado à natureza do curso desses participantes: por estarem imersos em um contexto no qual se dá enfoque na aplicação prática e técnica do conhecimento, engajarem-se em atividades de educação ambiental aplicada pode se aproximar mais de sua experiência real e cotidiana.

\section{Percepção da Gravidade das MCGs em Relação à Escala Espacial}

Como informado em método, os níveis da gravidade percebida das MCGs variavam em: muito grave, grave, não muito grave e não é grave, para a escala espacial nos níveis de meu bairro, minha cidade, meu país e mundo. Os resultados obtidos nessa questão se encontram representados graficamente na Figura 2.

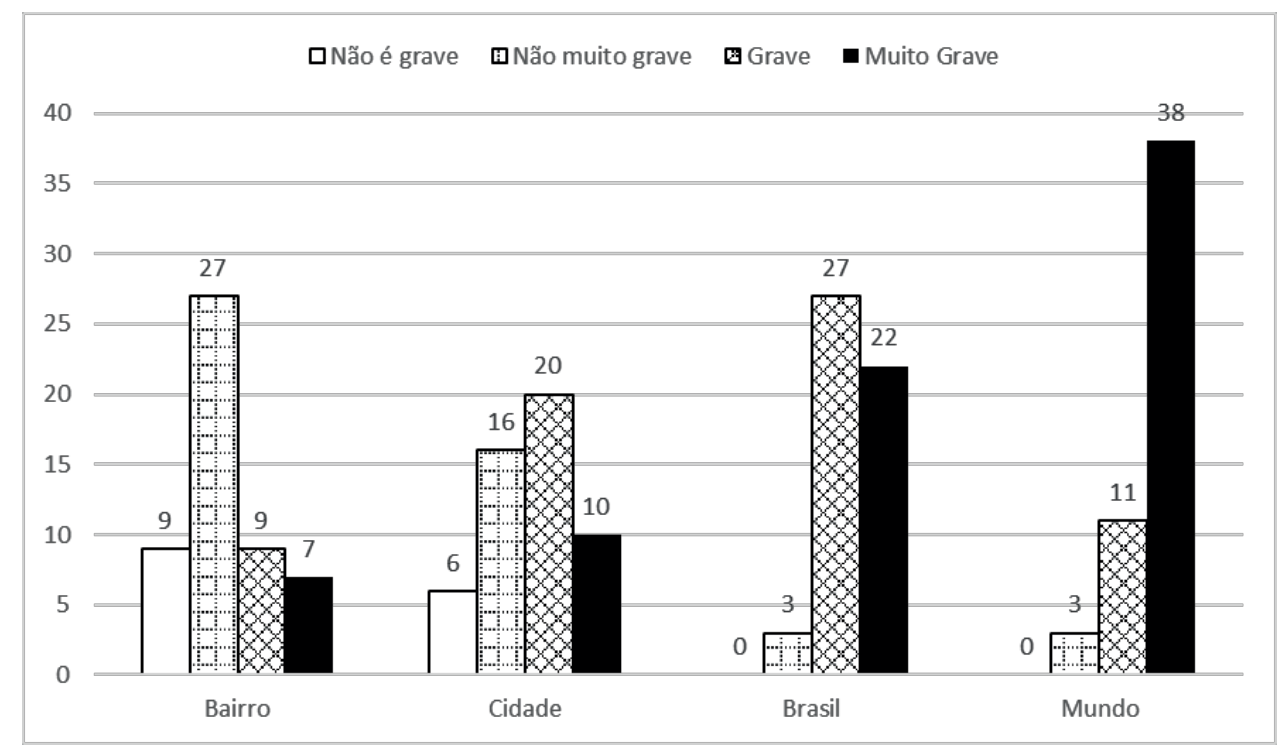

Figura 2. Percepção da gravidade das mudanças climáticas globais pelos níveis da escala espacial.

Na referida Figura, cada um dos quatro níveis da escala espacial (de bairro a mundo) agrupa os níveis de gravidade percebida das MCGs, expressos nas colunas (desde não é grave até muito grave). Percebe-se que a coluna de não é grave aparece apenas para bairro e cidade, desaparecendo para as duas escalas seguintes. O nível não muito grave, por sua vez, aparece em todas as escalas espaciais, mas seu decréscimo é notável à medida que a escala espacial se amplia; ou seja, são 27 respostas para bairro, mas apenas três para mundo. Os respondentes claramente atribuem menos gravidade às manifestações das MCGs nas escalas de bairro e cidade, nas quais ocorre sua vida cotidiana. Em tendência claramente oposta, o nível muito grave cresce consideravelmente a cada escala espacial, sendo notavelmente maior na escala de mundo (38 respostas) do que na de bairro (apenas sete).
Isto posto, constatamos que a gravidade do problema é, de fato, percebida, mas de forma viesada. Segundo as respostas, o problema existe, mas é consideravelmente mais presente para as escalas mais amplas (meu país e mundo) do que para as mais restritas (meu bairro e minha cidade), tendência que replica o que já fora encontrado em estudos anteriores (Gifford et al., 2009). Neles, foi verificada uma tendência em ver os problemas ambientais mais próximos do respondente de maneira mais favorável do que os problemas ambientais em escalas mais remotas - o viés de otimismo espacial. Uzzel (2000) havia, de maneira análoga, identificado como hipermetropia ambiental a visão embaçada que aplicamos às situações indesejáveis que estão próximas a nós. No já mencionado estudo de O'Neill (2008), essa constatação também se deu, acrescida do fato de que a ameaça das MCGs fora 
vista como mais ameaçadora para outros animais, que não os seres humanos.

\section{Quando as MCGs Constituirão uma Ameaça?}

A questão seguinte focalizava a temporalidade da ameaça das MCGs; ou seja, quando a situação é, ou passará a ser, grave. As respostas a ela estão esquematicamente representadas na Figura 3, cuja organização é semelhante à anterior. Nela, para cada nível da escala espacial (bairro, cidade, Brasil, mundo) consta a quantidade de respostas dos participantes relativa à proximidade temporal da ameaça das MCGs (conforme a legenda).

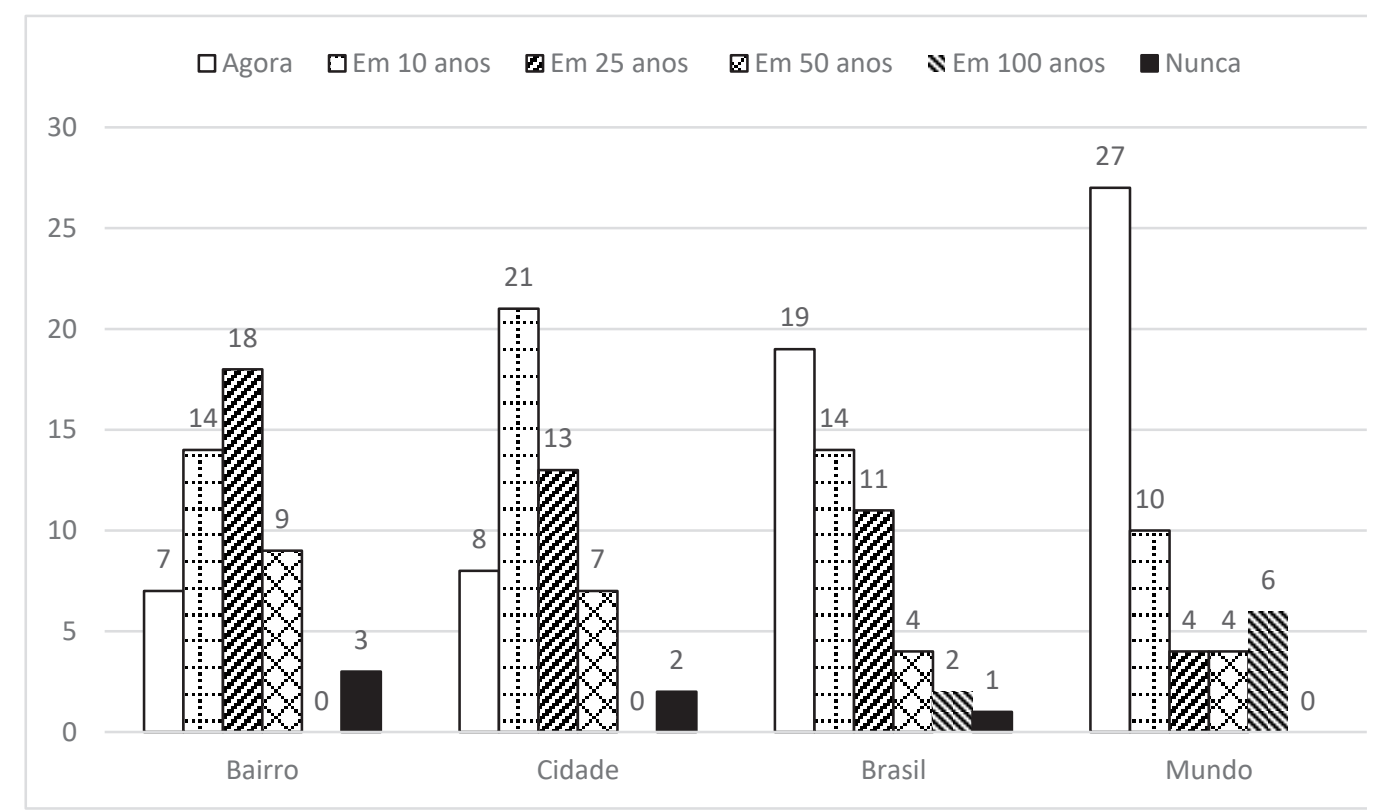

Figura 3. Percepção da época de ocorrência das mudanças climáticas globais, pelos níveis da escala espacial.

Assim, por exemplo, para a escala do país: 19 participantes responderam que a ameaça das MCGs já está ocorrendo, 14 avaliam que ela ainda demorará 10 anos, 11 consideram que acontecerá em 25 anos, quatro em 50 anos, dois somente em 100 anos e um respondente considera que esse perigo nunca irá ocorrer. Dito de outra maneira, para 44 respondentes (86\%) a ameaça das MCGs estará implantada no país em, no máximo, 25 anos.

Possivelmente o resultado que mais se destaca visualmente na Figura 3 é a coluna que representa o agora (ameaça imediata), consideravelmente mais alta para o mundo do que é para o bairro (27 e sete, respectivamente). Isso significa que, na percepção dos respondentes, as MCGs são percebidas como um problema real e atual e que tem presença quatro vezes mais expressiva na escala planetária do que na escala da vizinhança. Assim, o viés do otimismo espacial volta a se manifestar, aqui explicita e exclusivamente na escala temporal do imediato. As MCGs constituem um perigo concreto, que já está acontecendo; mas 46 participantes consideram que isso vale para país e mundo, enquanto apenas um terço dessa quantidade de respondentes (15) considera uma ameaça válida para os níveis de bairro e cidade. Para os dois espaços mais próximos do participante (bairro, cidade), e também para o nível do país, parece que a situação tende a melhorar (diminuição da ameaça) nas temporalidades mais longas/distantes, o que constituiria um otimismo também temporal.

Ao considerarmos o extremo oposto da escala proposta de temporalidades (em 100 anos e nunca), constatamos que talvez a era da negação da existência das MCGs esteja encerrada, ou quase, na opinião dos participantes. Esses dois níveis de temporalidade tiveram expressividade muito baixa, ou nula.

Os níveis intermediários de temporalidade (em 10, 25 ou 50 anos) não parecem indicar tendências claras, o que pode significar a influência de ao menos 
dois fatores. Um deles seria a incerteza - principalmente temporal - no que se refere às notícias e informações transmitidas sobre o assunto, dificultando que os respondentes se posicionem claramente sobre o tempo a decorrer até que os efeitos das MCGs possam ser mais objetivamente observáveis (reconhecidos, aceitos) pelo cidadão comum. $O$ outro fator diz respeito ao significado pessoal que esses intervalos de tempo assumem para cada um dos respondentes. Será que o intervalo de 10 anos, por exemplo, coloca as MCGs a uma "distância psicologicamente segura" do momento atual, ou já é próximo demais?

Seguindo com essa conjectura de distância psicologicamente segura, e levando em conta aquela hipótese de otimismo também temporal, é interessante observar nas respostas referentes às escalas de bairro, cidade e país, a diferença entre 25 e 50 anos. As colunas da Figura 3 mostram um "degrau" entre 25 e 50 anos, que tem a magnitude de aproximadamente o dobro de respostas para 25 anos, talvez em decorrência de este último intervalo ser psicologicamente mais próximo do respondente, asseguradamente dentro de seu tempo de vida. Contraditoriamente, há que se considerar a tendência social generalizada de valorização do imediatismo, da geração atual em detrimento das futuras e dos processos naturais que dão suporte à vida (Held, 2001). Portanto, nessa hipótese de um otimismo também temporal, qual temporalidade foi levada em conta pelo respondente, a da pessoa, a das MCGs, ou algum tipo de combinação das duas?

\section{Considerações finais}

A primeira constatação deste estudo diz respeito ao fato de que as pessoas parecem ter conhecimento de que as MCGs existem; parece estar superado o estágio de "negação geral" do problema, como mostra a ausência generalizada de escolha pelos níveis em 100 anos e nunca para a gravidade percebida nas MCGs (ver Figura 3). Entretanto, ainda há alguma indefinição ou confusão a respeito das estratégias de mitigação; em geral, as estratégias secundárias (mais características das formas tradicionais de enfrentamento dos problemas ambientais) ainda estão mais presentes no imaginário social do que aquelas diretamente relacionadas às MCGs.

Mesmo assim, nossa investigação fez uma constatação, até quanto sabemos, original, ao identificar três categorias de resposta como ação de mitigação das MCGs: consumo consciente, transporte e, principalmente, mudança do paradigma energético. Já que os participantes de nosso estudo constituíram, ao menos em parte, uma escolha nossa conveniente e premeditada e um recorte especializado da população, considerações mais amplas (generalização para além da população aqui estudada) sobre os resultados tornam-se inviáveis. Assim, é desejável que estudos ulteriores explorem sua ocorrência em outros setores da população geral, para verificar a presença dos sinais aqui detectados de uma visão mais bem informada e mais amplamente crítica, possivelmente baseada na noção de sustentabilidade, que talvez esteja emergindo em nosso meio.

Constatamos a ocorrência do viés do otimismo espacial (e.g., Clayton et al., 2015; Gifford, 2011; Gifford et al., 2009), que diz respeito à dificuldade de perceber (ou de admitir?) a ameaça representada pelas MCGs à medida que a escala espacial torna-se menos abrangente e, por isso, mais próxima da pessoa e mais concreta. Essa constatação traz em seu bojo um paradoxo geral e alguns questionamentos.

Na Figura 2, a ameaça das MCGs é considerada como muito grave em muito maior frequência para o mundo do que para o bairro $(38>7)$. Similarmente, na Figura 3 as MCGs são percebidas como um perigo imediato na escala global, mas para o bairro a quantidade de respostas é a quarta parte $(27>7)$. Como é possível que a ameaça seja real e iminente para a escala global, sem que se admita sua concomitante expressão em escala local? A "lógica" envolvida na explicação desse paradoxo tem contornos inevitavelmente psicológicos e precisa continuar a ser investigada em pesquisas futuras.

Seria esse viés uma deficiência no conhecimento das MCGs propriamente dito? Como lidar adequadamente com uma coisa que não se conhece direito? Se as pessoas se apropriam ou não da informação, e como o fazem, ainda é discutível, mas também é preciso nos questionarmos a respeito da natureza e qualidade da informação que é comunicada sobre MCGs (e.g., Moser \& Dilling, 2006), já que necessariamente esse conhecimento se baseia em formas indiretas (de segunda mão) de comunicação sobre o fenômeno.

$E$, para além da dimensão puramente cognitiva, é preciso considerar o matiz afetivo com que essas informações são carregadas e transmitidas. Parece razoável supor que essa diferenciação local-global se relacione ao panorama divulgado pelos meios de comunicação, que costumam enfatizar um cenário de "desastre", quase sempre limitado ao plano global, planetário (O'Neill \& Nicholson-Cole, 2009; Pinheiro \& Farias, 
2015). Ao mesmo tempo, existe a possibilidade de que os ambientes locais sofram uma modulação afetiva relacionada a características da própria pessoa envolvida, já que parte do que somos é constituída pelos ambientes em que já vivemos (identidade de lugar). A motivação individual por manter uma auto imagem positiva pode implicar em que não se perceba ou admita problemas na imagem do lugar imediato em que se está inserido (Gifford et al., 2009). Para apaziguar a dissonância, aceita-se que o problema exista, mas apenas "lá fora", na escala global (em relação ao qual o indivíduo nada pode fazer) e não atinge ainda o espaço concreto e imediato de vida desse indivíduo; consequentemente, não o atinge, não o mobiliza, permitindo que ele/ela siga se sentido confortável.

A importância de se estudar as formas percebidas de mitigação das MCGs e os vieses associados à percepção desse fenômeno, otimistas ou pessimistas, reside principalmente no caráter aplicado dessa área de investigação científica. Afinal, o objetivo último desses esforços é o planejamento e a implementação de estratégias que promovam o crescente engajamento da população no enfrentamento às MCGs. Entender como o indivíduo recebe, ou não, a informação e se engaja, ou não, em alguma transformação cotidiana é um dos grandes desafios - pouco explorado - da comunicação científica, particularmente em se tratando das MCGs.

Reconhecemos a rede multicultural e internacional de conflitos que cercam o tema; assim, nosso propósito não é de responsabilização única do indivíduo. Todavia, este pode se informar sobre tais temáticas e agir em consonância, seja no apoio a políticas públicas que busquem mitigar as MCGs, na cobrança ou construção destas, ou ainda, no engajamento direto ou indireto em ações tradicionais de proteção ambiental. Nesse sentido, as condições sociais e as condições de existência das pessoas precisam ser levadas em conta por programas de educação ambiental ou iniciativas governamentais que venham a ser implantadas, para que o engajamento em ações de mitigação seja estimulado, mas dentro de uma atuação possível, alcançável e local.

\section{Agradecimentos}

Ao apoio do CNPq, na forma de bolsa de produtividade em pesquisa e apoio a projeto de pesquisa (Edital Universal) para o primeiro autor, de iniciação científica para a segunda autora e ao apoio da CAPES, pela bolsa de doutorado da terceira autora.

\section{Referências}

Bardin, L. (1977). Análise de conteúdo. Lisboa: Edições 70.

Barros, H. C. L. (2011). Mudanças climáticas globais e o compromisso pró-ecológico de adolescentes natalenses (Dissertação de Mestrado). Recuperado de https://repositorio.ufrn.br/jspui/ handle/123456789/17481

Barros, H. C. L., \& Pinheiro, J. Q. (2013). Dimensões psicológicas do aquecimento global conforme a visão de adolescentes brasileiros. Estudos de Psicologia, 18(2), 173-182. doi: 10.1590/ S1413-294X2013000200002

Bauer, M. W. (2002). Análise do conteúdo clássica: uma revisão. In M. W. Bauer \& G. Gaskell (Orgs.), Pesquisa qualitativa com texto, imagem e som (P. Guareschi, Trad., pp. 189-217). Petrópolis: Vozes.

Breakwell, G. M. (2010). Models of risk construction: Some applications to climate change. Wiley Interdisciplinary Reviews: Climate Change, 1(6), 857-870. doi: 10.1002/wcc.74

Castro, T. G., Abs, D., \& Sarriera, J. C. (2011). Análise de conteúdo em pesquisas de psicologia. Psicologia, Ciência e Profissão, 4, 814-825. doi: 10.1590/S1414-98932011000400011

Clayton, S., Devine-Wright, P., Stern, P. C., Whitmarsh, L., Carrico, A., Steg, L., ... Bonnes, M. (2015). Psychological research and global climate change. Nature Climate Change, 5(7), 640-646. doi:10.1038/ nclimate2622

Corral-Verdugo, V. (2010). Psicologia de la Sustentabilidad, un análisis de lo que nos hace pro-ecológicos y pro-sociales. México, DF.: Trillas.

Doherty, T. J., \& Clayton, S. (2011). The psychological impacts of global climate change. American Psychologist, 66(4), 265-276. doi: 10.1037/a0023141

Feitelson, E. (1991). Sharing the globe: The role of attachment to place. Global Environmental Change, 1(5), 396-406. doi: 10.1016/0959-3780(91)90005-E

Gifford, R. (2008). Psychology's essential role in alleviating the impacts of climate change. Canadian Psychology/Psychologie Canadienne, 49(4), 273-280. doi: 10.1037/a0013234

Gifford, R. (2011). The dragons of inaction: psychological barriers that limit climate change mitigation and adaptation. American Psychologist, 66, 290-302. doi: $10.1037 / \mathrm{a} 0023566$

Gifford, R. , Scannell, L. , Kormos, C. , Smolova, L. , Biel, A. , Boncu, S. , ... Uzzell, D. (2009). Temporal pessimism and spatial optimism in environmental assessments: An 18-nation study. Journal of Environmental Psychology, 29(1), 1-12. doi 10.1016/j. jenvp.2008.06.001

Held, M. (2001). Sustainable development from a temporal perspective. Time \& Society, 10(2/3), 351-366. doi: 10.1177/0961463X01010002011

Intergovernmental Panel On Climate Change (2014). Climate Change 2014: Synthesis Report. Genebra: Autor.

Leiserowitz, A. (2005). American risk perceptions: Is climate change dangerous? Risk Analysis, 25, 1433-1442. doi: 10.1111/j.1540-6261.2005.00690.x

Lima, G. F. C. (2009). Mudanças climáticas e conservação social: riscos do aquecimento global. Gaia Scientia, 3(1), 35-46. Recuperado de http:// www.periodicos.ufpb.br/ojs/index.php/gaia/article/view/3342/2743

Lorenzoni, I., Pidgeon, N. F., \& O'Connor, R. E. (2005). Dangerous climate change: The role for risk research. Risk Analysis, 25(6), 1387-1398. doi: 10.1111/j.1539-6924.2005.00686.x

Moser, G., \& Uzzel, D. (2004). Psychology and the challenge of global environmental change. IHDP UPDATE [Newsletter of the 
International Human Dimensions Programme on Global Environmental Change], 4, 1-2.

Moser, S. C., \& Dilling, L. (2006). (Orgs.). Creating a climate for change communicating climate change and facilitating social change. Nova York: Cambridge University Press.

Nobre, C., Marengo, J. A., Soares, W., Assad, E., Schaeffer, R., Scarano, F. R., \& Hacon, S. S. (2016). Riscos de mudanças climáticas no Brasil e limites à adaptação. Brasília: Embaixada do Reino Unido no Brasil.

O'Neill, S. J. (2008). An iconic approach to communicating climate change (Tese de doutorado). Recuperado de https://crudata.uea.ac.uk/cru/ pubs/thesis/2008-oneill/thesis.pdf

O' Neill, S. J., \& Nicholson-Cole, S. (2009). "Fear won't do it”: promoting positive engagement with climate change through visual and iconic representations. Science Communication, 30, 355-379. doi: 10.1177/1075547008329201

Pawlik, K. (1991). The psychology of global environmental change: some basic data and an agenda for co-operative international research. International Journal of Psychology, 26, 547-563. doi 10.1080/00207599108247143

Painel Brasileiro de Mudanças Climáticas (2014a). Base científica das mudanças climáticas. Contribuição do Grupo de Trabalho 1 do Painel Brasileiro de Mudanças Climáticas ao Primeiro Relatório da Avaliação Nacional sobre Mudanças Climáticas [T. Ambrizzi, \& M. Araújo, (Orgs.)]. Rio de Janeiro: COPPE, Universidade Federal do Rio de Janeiro.

Painel Brasileiro de Mudanças Climáticas (2014b). Impactos, vulnerabilidades e adaptação às mudanças climáticas. Contribuição do Grupo de Trabalho 2 do Painel Brasileiro de Mudanças Climáticas ao Primeiro Relatório da Avaliação Nacional sobre Mudanças Climáticas [E. D. Assad, \& A. R. Magalhães (Orgs.)]. Rio de Janeiro: COPPE, Universidade Federal do Rio de Janeiro.

Painel Brasileiro de Mudanças Climáticas (2014c). Mitigação das mudanças climáticas. Contribuição do Grupo de Trabalho 3 do Painel Brasileiro de Mudanças Climáticas ao Primeiro Relatório da Avaliação
Nacional sobre Mudanças Climáticas [M. M. C. Bustamante \& E. L. L. Rovere (Orgs.)]. Rio de Janeiro: COPPE, Universidade Federal do Rio de Janeiro.

Pinheiro, J. Q., \& Farias, A. C. (2015). In search of a positive framework for communications about Global Climate Change. Psyecology, 6, 229-251. doi: 10.1080/21711976.2015.1026084

Renn, O. (2011). The social amplification/attenuation of risk framework: application to climate change. Wiley Interdisciplinary Reviews: Climate Change, 2(2), 154-169. doi: 10.1002/wcc.99

Rosa, L. P. (2008). Apresentação. In R. Schaeffer, A. S. Szklo, A. F. P. Lucena, R. R. Souza, B. S. M. C. Borba, I. V. L. Costa, ... S. H. F. Cunha (Orgs.), Mudanças climáticas e segurança energética no Brasil [Sumário Executivo] (pp. 6-8). Rio de Janeiro: Universidade Federal do Rio de Janeiro, COOPE.

Scannell, L., \& Gifford, R. (2013). Personally relevant climate change:The role of place attachment and local versus global message framing in engagement. Environment \& Behavior, 45, 60-85.

Stern, P. C., Young, O. R., \& Druckman, D. (1992). Global environmental change: Understanding the human dimensions. Washington: National Academy of Sciences.

Swim, J., Clayton, S., Doherty, T., Gifford, R., Howard, G., Reser, J., ... Weber, E. (2009). Psychology and global climate change: Addressing a multi-faceted phenomenon and set of challenges. Washington: American Psychological Association.

Uzzell, D. (2000). The psycho-spatial dimension of global environmental problems. Journal of Environmental Psychology, 20(4), 307-318. doi: 10.1006/jevp.2000.0175

Whitmarsh, L. (2009). Behavioural responses to climate change: Asymmetry of intentions and impacts. Journal of Environmental Psychology, 29, 13-23. doi: 10.1016/j.jenvp.2008.05.003

Whitmarsh, L., O'Neill, S., \& Lorenzoni, I. (2011). (Orgs.). Engaging the public with climate change: Behaviour change and communication. Londres: Earthscan.

José Q. Pinheiro, Doutor em Psicologia Ambiental pela Universidade do Arizona (UA), Tucson, EUA, é Professor Titular aposentado e Colaborador voluntário no Programa de Pós-graduação em Psicologia da Universidade Federal do Rio Grande do Norte (UFRN). Endereço para correspondência: Av. Estrela do Mar, 2263, Natal, RN, CEP 59.090-400.

Telefone: (84) 3219-3366. E-mail: pinheiro@cchla.ufrn.br

Giselli R. C. Cavalcanti, Psicóloga pela Universidade Federal do Rio Grande do Norte (UFRN), é Aluna de mestrado no Programa de Pós-graduação em Psicologia na Universidade Federal do Rio Grande do Norte (UFRN). E-mail: giselliccruz@gmail.com

Hellen C. L. Barros, Doutora em Psicologia no Programa de Pós-graduação em Psicologia pela Universidade Federal do Rio Grande do Norte (UFRN), é Professora do curso de Psicologia da Universidade Potiguar - Laureate International Universities (UnP). E-mail: hellenwbarros@hotmail.com 